\title{
INFERENCIAS PALEODEMOGRÁFICAS EN EL NOROESTE DE PATAGONIA A PARTIR DEL SITIO AQUIHUECÓ, PROVINCIA DEL NEUQUÉN, ARGENTINA
}

\author{
PALEODEMOGRAPHIC INFERENCES OF THE NORTHWEST OF PATAGONIA. \\ INSIGHTS FROM THE AQUIHUECÓ SITE, NEUQUÉN PROVINCE, ARGENTINA
}

\author{
Florencia Gordón $n^{1,2}$, Marien Béguelin ${ }^{1,3}$, Paula Novellino ${ }^{1,4}$ y Fernando Archuby ${ }^{1,3}$
}

\begin{abstract}
En este trabajo se presenta la estructura sexo-etaria del sitio Aquihuecó y se discuten sus implicancias para la dinámica poblacional del noroeste de Patagonia argentina a comienzos del Holoceno Tardío. El sitio se encuentra emplazado en un médano sobre el Río Curí Leuvú (Prov. del Neuquén) y ha sido definido como un sitio de entierro con una cronología que abarca desde $4304 \pm 59$ hasta $3650 \pm 70$ años AP. Se estimó un número mínimo de individuos de 64, incluyendo elementos desarticulados y fragmentarios de superficie y se confeccionó una curva de edades de muerte en base a los 43 esqueletos hallados in situ. Los resultados indican la representación de ambos sexos y todas las categorías de edad, excepto el rango 15-19,9 años. La curva está caracterizada por un patrón bimodal con dos picos correspondientes a los rangos 0-4,9 años $(20,92 \%)$ y 25-29,9 (11,63\%). Se calculó la proporción juveniles/adultos y la mortalidad promedio en la niñez con el fin de caracterizar la dinámica poblacional. Ambos índices señalan que se trata de una muestra sesgada aunque en base a otras líneas (i.e. molecular, radiocarbónica, isotópica y zooarqueológica) se sugiere que podría corresponder a una población creciente. La curva se comparó con otras obtenidas para sitios de áreas vecinas. Si bien el patrón general es similar, no se encontraron correlaciones estadísticamente significativas.
\end{abstract}

Palabras claves: Noroeste de Patagonia, Holoceno Tardío, paleodemografía, cazadores-recolectores, bioarqueología.

In this paper we present the sex and age-at-death structure of the Aquihuecó archaeological burial site and discuss its implications for the population dynamics of northwestern Argentinian Patagonia at the beginning of the Late Holocene. The site is located on a sand-dune in the Curi Leuvú River valley (Neuquén Province) and has been defined as a burial site with a chronology that spans from $4304 \pm 59$ to $3650 \pm 70$ years BP. A minimum number of 64 individuals was estimated, including fragmentary bone remains spread on surface, and a mortality curve was built based on 43 skeletons in situ. Both sexes and all age categories, except the range 15-19.9 years, are represented in the sample. The mortality curve is characterized by a bimodal pattern with two peaks corresponding to the ranges 0-4.9 years (20.92\%) and 25-29.9 (11.63\%). The Juvenile/Adult Ratio and the Mean Childhood Mortality were calculated in order to characterize the population dynamics. Although both indices indicate a biased sample, other evidences (i.e. molecular, radiocarbon, isotopic and zooarchaeological) suggest it is growing population. The mortality curve was compared with those obtained for sites located in neighboring areas. The pattern is similar, but no statistically significant correlations were found.

Key words: Northwestern Patagonia, Late Holocene, paleodemography, hunter-gatherers, bioarchaeology.

El sitio Aquihuecó fue detectado en el año 1997 y como resultado de las sucesivas investigaciones se generó un cúmulo de información que actualmente permite caracterizar al sitio en términos paleodemográficos. Si bien en Norpatagonia existen entierros con la misma o incluso mayor antigüedad (p.ej., Cantera de Rodados Villalonga, Tres Bonetes 1, Hermanos Lazcano, entre otros -Della Negra et al. 2014; Martínez 2017; Martínez

\footnotetext{
${ }^{1}$ CONICET Consejo Nacional de Investigaciones Científicas y Técnicas, Buenos Aires, Argentina.

2 Laboratorio Anexo del Museo de La Plata (FCNyM, UNLP). La Plata, Prov. de Buenos Aires, Argentina. fgordon2007@ gmail.com

${ }^{3}$ Instituto de Investigación en Paleobiología y Geología (UNRN). Prov. de Río Negro, Argentina. mbeguelink@gmail.com; farchuby@gmail.com

${ }^{4}$ Museo de Ciencias Naturales y Antropológicas “J. C. Moyano”. Mendoza, Prov. de Mendoza, Argentina. paunove@ gmail.com
} 
y Flensborg 2018), la singularidad de Aquihuecó reside en el elevado número de individuos inhumados en un espacio acotado correspondiente a la transición Holoceno Medio - Holoceno Tardío (Della Negra y Novellino 2005; Della Negra et al. 2009; Gordón et al. 2019) lo que lo convierte en un sitio relevante para comprender la dinámica poblacional del pasado. El norte neuquino constituye un área clave desde un punto de vista paleodemográfico ya que geográficamente conecta diversas regiones o sub-regiones a través de grandes ríos y pasos bajos (i.e. Cuyo, Pampa, Patagonia meridional, NE de Patagonia y Chile) y ecológicamente zonas con características de diversas provincias (i.e. Provincias del Monte, Patagónica y Altoandina). Asimismo, existe evidencia que sugiere contactos intergrupales y una serie de prácticas culturales que habrían ocurrido en momentos anteriores que en otras áreas (Della Negra 2008; Della Negra y Novellino 2005; Della Negra et al. 2009, 2014; Hajduk et al. 2007; Hajduk y Vargas 2017; Lema et al. 2012; Perez et al. 2009).

Hasta el momento se estudiaron aspectos relacionados con la morfología corporal (Béguelin 2017; Cobos y Bernal 2017; Perez et al 2009), la dieta (Gordón et al. 2013, 2018b), la salud bucal (Gordón y Novellino 2017), las técnicas de procesamiento de los alimentos (Lema et al. 2012; Rindel 2017) y el ajuar funerario (Della Negra e Ibañez 2012) así como procesos evolutivos y ecológicos a los que estuvieron sujetas estas poblaciones (Gordón et al. 2018; Perez et al. 2016). Sin embargo, los aspectos paleodemográficos no se han abordado de manera integrada. Con la totalidad del material recuperado y considerando que en el sitio ya no quedarían individuos, o al menos no una cantidad que pueda aumentar significativamente la muestra, es posible realizar una reconstrucción desde esta perspectiva.

Los análisisdemográficosocupanunlugarcentralen las investigaciones antropológicas en tanto constituyen estudios cuantitativos de las poblaciones. La demografía estudia los procesos que determinan la conformación, perduración y desaparición de las poblaciones, tales como fecundidad, mortalidad y migración (LiviBacci 1993). Dado que los análisis demográficos incluyen las escalas espaciales y temporales requeridas para la ocurrencia de esos cambios, la perspectiva paleodemográfica es particularmente importante en los estudios bioarqueológicos (Chamberlain 2006). La paleodemografía, cuyo desarrollo fue relevante durante los últimos 50 años, no estuvo exenta de críticas y reformulaciones debido a los sesgos y dificultades presentadas en el proceso inferencial (Bocquet-Appel y Masset 1982; Sattenspiel y Harpending 1983). Sin embargo, una serie de esfuerzos teórico-metodológicos, dirigidos al mejoramiento de las técnicas de estimación de sexo y edad (p.ej., técnicas de regresión para métodos de edad, estimadores paleodemográficos, métodos de máxima probabilidad y modelos bayesianos, entre otros), permitieron mejorar las interpretaciones (Chamberlain 2006; Hoppa 2001; Hoppa y Vaupel 2002; Milner et al. 2000).

En este contexto, el sitio Aquihuecó reviste una gran relevancia, tanto para el estudio de los cazadoresrecolectores de Patagonia en general como para los grupos de Norpatagonia en particular, debido a la cantidad de individuos inhumados en un médano de dimensiones acotadas (18 $\mathrm{m} \times 35 \mathrm{~m}$, aproximadamente) y a su ubicación geográfica y cronológica.

\section{Breve Historia de las Investigaciones en Aquihuecó y Características del Sitio}

El sitio de entierro Aquihuecó se emplaza en la porción superior de un médano ubicado en $37^{\circ}$ $05^{\prime} 3,5^{\prime \prime} \mathrm{S}$ y $70^{\circ} 22^{\prime} 31^{\prime \prime} \mathrm{O}$, a unos $500 \mathrm{~m}$ de la margen izquierda del Río Curi Leuvú y a 1170 msnm (Dto. Chos Malal, provincia del Neuquén) (Figura 1a y b). En marzo de 1997, producto de una denuncia realizada por pobladores del área, se realizaron las primeras prospecciones y recolección superficial de restos óseos en un radio de $100 \mathrm{~m}$, estimándose un número mínimo de individuos NMI=4. Se determinó que uno de ellos era adulto mientras que los tres restantes eran subadultos. Con el objetivo de obtener un primer fechado radiocarbónico se tomó una muestra ósea del perfil norte a $1,90 \mathrm{~m}$ de profundidad y se obtuvo una edad de 3650 \pm 70 años AP (Della Negra y Novellino 2005). Este fechado no se presenta en la tabla de fechados dado que se desconoce a qué individuo corresponde. En el año 2003 el Escuadrón 30 de Gendarmería Nacional de Chos Malal realizó una denuncia a partir de la exposición de huesos en el perfil norte del médano. En esa oportunidad se realizó una nueva recolección superficial y se excavó sistemáticamente un sector del sitio. Se estimó un NMI=28 (16 adultos y 12 subadultos) (Della Negra y Novellino 2005). En los años 2006 y 2007 se realizaron nuevas prospecciones y excavaciones en las que se identificaron 15 entierros. El NMI se estimó provisoriamente en 56 individuos (30 adultos y 26 subadultos) (Della Negra et al. 2009). Asociados a los esqueletos se hallaron molinos y manos de moler, cuentas de valvas, cantos rodados de entre 10 y $30 \mathrm{~cm}$, instrumentos con filo, puntas de proyectil y preformas (Della Negra y Novellino 2005). Asimismo, se identificaron piedras que demarcaban los entierros, tanto lajas horizontales como rocas andesíticas de fractura natural trapezoidal dispuestas de manera vertical (Figura 2a y b) (Della Negra e Ibáñez 2012; Della Negra et al 2009). En el año 2012 se realizó una excavación en la que se recuperaron 16 individuos in situ y una gran cantidad de restos óseos humanos en 
superficie con grados variables de meteorización. Previo al inicio de las tareas de excavación, la dispersión de los fragmentos de hueso, incluso de huesos completos, se extendía $42 \mathrm{~m}$ en dirección sur (Figura 3). En la última campaña de excavación (febrero de 2017) se recuperaron tres individuos por debajo de los niveles anteriores, dos adultos masculinos y un subadulto de sexo indeterminado. El último individuo hallado (Ind. 43), emplazado en los niveles inferiores, presentaba una modalidad de entierro diferente a las registradas hasta el momento. Una acumulación de rocas de tamaños entre 15 y más de $45 \mathrm{~cm}$ de diámetro se disponían en círculo por encima del individuo (Figura 2c). Hasta el momento, se realizaron siete fechados radiocarbónicos sobre los restos humanos que se suman al ya mencionado (Gordón et al. 2019; Lema et al. 2012). Sobre la base de las dataciones se estimó un rango de uso de $654 \pm 129$ años. Si bien el médano no fue excavado en su totalidad, se realizaron sondeos en el sector restante así como en la base de la excavación, con resultados negativos.

En este trabajo se integra la totalidad de los individuos recuperados en un análisis paleodemográfico tendiente a interpretar la dinámica poblacional del noroeste patagónico que incluye tanto a los individuos hallados in situ como al material óseo humano recolectado en superficie.

\section{Materiales y Métodos}

El material analizado corresponde a los esqueletos recuperados en las campañas de prospección y excavación y se encuentra alojado en la Subsecretaría de Cultura, Juventud y Deporte de la Provincia del Neuquén y en el Museo "Ana M. Biset" en el paraje Caepe Malal (Ley Provincial No 2.184/96).

Para la estimación del sexo, en los adultos se observó la morfología del coxal (i.e. región subpúbica), la amplitud de la escotadura ciática mayor y la presencia de surco preauricular (Buikstra y Ubelaker 1994; Phenice 1969). También se tomaron en cuenta indicadores morfológicos del cráneo (i.e. cresta nucal, proceso mastoideo, margen supra orbital, glabela y eminencia del mentón) (Acsadi y Nemeskeri 1970; Buikstra y Ubelaker 1994). Otros rasgos considerados fueron los diámetros máximos de la cabeza de fémur y húmero (Béguelin y González 2008; Béguelin et al. 2011). En los subadultos el sexo se estimó en base a indicadores morfológicos del ilion (i.e. ángulo de la escotadura ciática, criterio del arco, curvatura de la cresta ilíaca, elevación de la superficie auricular) y la mandíbula (i.e. prominencia del mentón, forma del arco dental anterior, eversión del gonion, ángulo mandibular) (Loth y Henneberg 2001; Luna 2008; Molleson et al. 1998; Schutkowski 1993; Weaver 1980).
Para la estimación de la edad de muerte de los individuos adultos se consideraron los cambios morfológicos de la sínfisis púbica(Brook y Suchey 1990) y los cambios en la superficie auricular y retroauricular del ilion (Lovejoy et al. 1985; Meindl y Lovejoy 1989). Asimismo, se siguió el sistema latero-anterior de cierre de suturas craneanas (Meindl y Lovejoy 1985) con el fin de ubicar a cada individuo en categorías amplias (i.e. adulto joven-20 - 34,9 años-, medio -35 - 49,9 añosy senil - >50 años-) cuando no fue posible definir rangos de edad más acotados y en particular para comparar la distribución etaria de Aquihuecó con la de otros sitios. Finalmente, se observó el estado de los elementos de fusión tardía (i.e. epífisis de clavículas, cresta ilíaca y sincondrosis basioccipital) (Kahana et al. 2003; Owin Webb y Suchey 1985; Schulze et al. 2006). La estimación de la edad en subadultos se llevó a cabo mediante la secuencia de formación y calcificación dental, elementos de fusión temprana (i.e. centros de osificación primaria en pelvis, vértebras y occipital) y fusión de epífisis y procesos de diversos elementos (Anderson et al. 1976; Demirjian et al. 1973; Lewis 2007; McKern y Stewart 1957; Scheuer y Black 2004; Ubelaker 1982, entre otros). Se consideraron subadultos a los individuos $<20$ años.

Se calculó el Número Mínimo de Individuos (NMI) como estimación de base para reconstruir el perfil demográfico (Mengoni-Goñalons 1999; Ubelaker 1974). Se contabilizó una serie de huesos o zonas diagnósticas de los elementos de la muestra total (i.e. individuos enterrados + material de superficie). Para la estimación del NMI no se consideró el sexo, debido a la gran cantidad de elementos de superficie y al elevado grado de fragmentación y/o meteorización que presentaban ciertos restos. Asimismo, la división etaria para el cálculo del NMI solo distinguió adultos de subadultos en base al tamaño de los elementos y la fusión de epífisis.

Para calcular el NMI se contabilizó la frecuencia de los siguientes elementos por lateralidad: mastoides, cavidad glenoidea de la escápula, rótula, calcáneo, astrágalo, ilion, isquion, pubis y mandíbula. En los huesos largos se distinguieron regiones proximales y distales (medial y lateral en la clavícula), en húmero, cúbito, radio, clavícula, fémur, tibia y peroné. Asimismo, se contabilizaron las vértebras $\mathrm{C} 1$ y $\mathrm{C} 2$. En todos los casos se consideró la presencia del elemento cuando estaba representado en más del $75 \%$ para asegurar que no se computara dos veces un individuo.

A partir de las estimaciones de sexo y edad de la muestra de individuos enterrados se construyó la curva de mortalidad tomando intervalos de cinco años (i.e. $0-4,9 ; 5-9,9 ; 10-14,9 ; 15-19,9 ; 20-24,9 ; 25-$ 29,9 ; 30-34,9; 35-39,9; 40-44,9; 45-49,9; >50 años). 

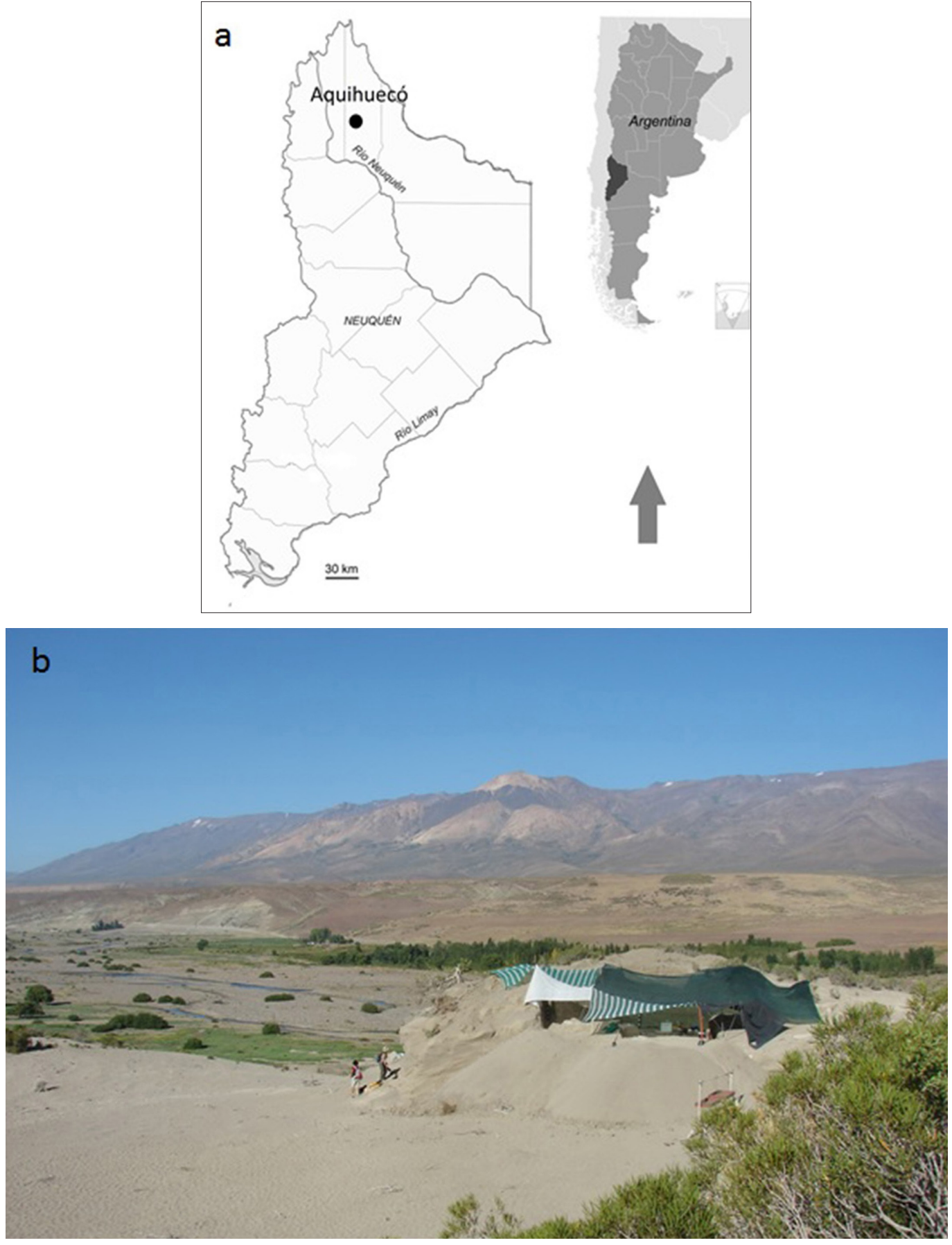

Figura 1. Aquihuecó: (a) ubicación geográfica, (b) vista del médano desde el este, abajo se observa el valle del río Curi Leuvú y hacia el oeste (en frente) la Cordillera del Viento.

Aquihuecó: (a) geographic location, (b) view from the east side of the sand-dune; in the middle the Curi Leuvú River Valley and in the background, westward, the Cordillera del Viento. 


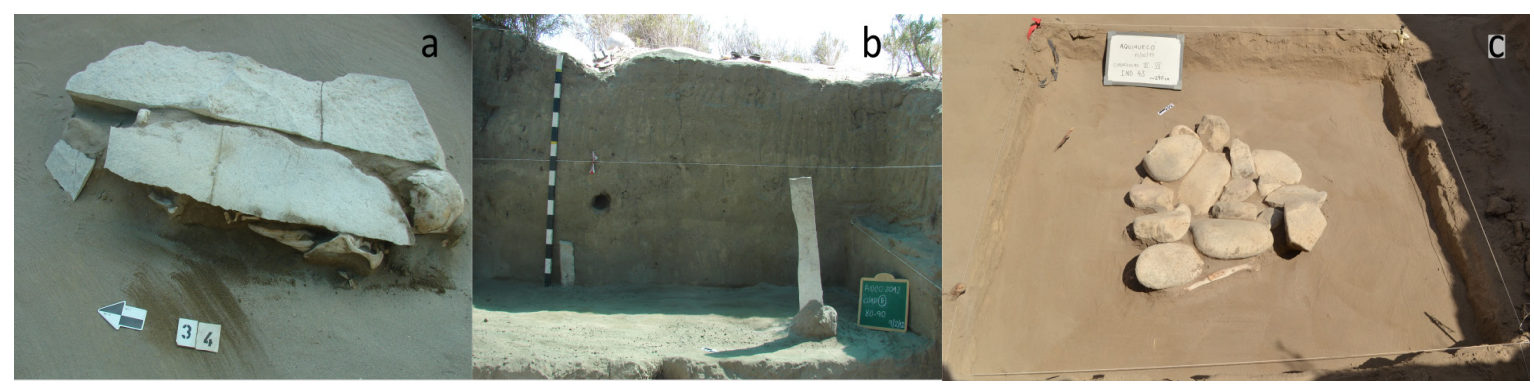

Figura 2. Modalidades de entierro registradas en Aquihuecó. (a) Lajas horizontales sobre el individuo, (b) roca andesítica vertical señalando un entierro, (c) montículo de rocas por encima de un individuo.

Burial modalities registered in Aquihuecó. (a) Horizontal slabs over a single burial, (b) vertical andesitic marking a burial, (c) stone mound over a single burial.
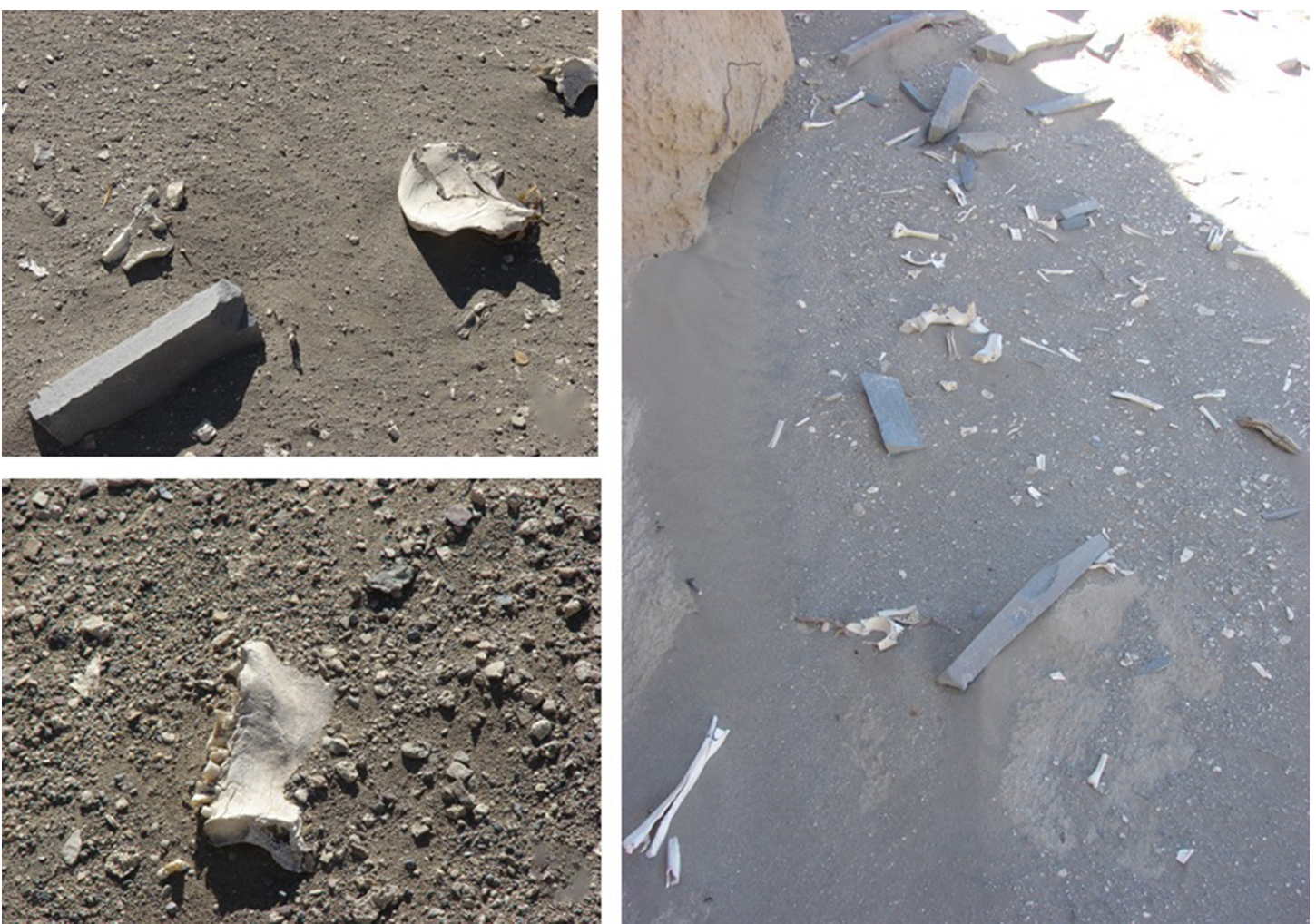

Figura 3. Dispersión de material óseo humano hallado en la superficie del médano (febrero de 2012).

Human bone remains scattering on the sand-dune surface (February 2012). 
Esta amplitud contiene la mayor parte del error en la determinación de la edad y a su vez es lo suficientemente acotada para discernir patrones de mortalidad (Bernal et al. 2004; Chamberlain 2006; Ubelaker 1989). Con el fin de comparar la curva de Aquihuecó con las de otros sitios se seleccionaron muestras de Patagonia meridional (Sierra Colorada (SAC), noroeste de Santa Cruz, García Guraieb 2010; García Guraieb et al. 2007; 2015), noreste de Patagonia (Paso Alsina I (PA); Flensborg 2012; Flensborg et al. 2015), Pampa Seca (Lihué Calel, Chenque I (LC), Luna 2008, 2012) y Cuyo (B6, Novellino et al. 2013). Asimismo, se consideró una muestra independiente procedente de un sitio ubicado en un área externa cuya base de subsistencia es diferente a la del resto de los sitios considerados (Punta Teatinos, desierto semiárido de Chile (PT), Quevedo et al. 2000). En el caso de Sierra Colorada se consideró la muestra tardía por tratarse, probablemente, de una unidad demográfica diferente a las muestras tempranas (García Guraieb, com. pers. 2018).

Para realizar dicha comparación las categorías de subadultos se mantuvieron con intervalos de cinco años. Cuando alguna categoría abarcaba dos de las aquí definidas los individuos fueron repartidos equitativamente. Los adultos fueron agrupados en tres categorías amplias (i.e. adultos jóvenes, medios y seniles). Siguiendo a García Guraieb (2010), con el objetivo de maximizar la información disponible, aquellos individuos adultos que no pudieron ser asignados a rangos más específicos fueron repartidos proporcionalmente entre las demás categorías, según la representación del perfil creado con los individuos de edad asignada.

Finalmente, se estimaron los siguientes parámetros denominados "proporciones de muerte" (death ratios):

(a) La relación entre la cantidad de juveniles (514,9 años) y adultos (+20 años) del siguiente modo: JAR (juvenile/adult ratio $)=\mathrm{n}_{\text {juveniles }} / \mathrm{n}_{\text {adultos }}$. Los rangos etarios considerados permiten minimizar la subestimación de individuos menores a los cinco años como así también las dificultades metodológicas para estimar edad de muerte en adultos (Jackes 1986, 1992). Según Jackes (2011) valores por encima de 0,38 sugieren un sesgo en la muestra mientras que aquellos por debajo de ese umbral permiten interpretar la dinámica poblacional.

(b) La mortalidad promedio en los individuos juveniles (Mean Childhood Mortality o MCM, Jackes 1986, 2011). Este parámetro se refiere al promedio de la probabilidad de muerte $(q x)$ de las tres categorías de juveniles (5-9,9; 10-14,9 y 15-19,9 años) (BocquetAppel y Masset 1977, 1982). La qx de cada categoría de edad se calculó como el cociente entre el porcentaje de muertes y el índice de supervivencia para ese rango etario (Ubelaker 1989). En este caso el umbral por encima del cual las muestras estarían sesgadas se ubica en 0,135 (Jackes 2011).

El enfoque basado en estimadores del tipo "proporciones de muerte" admite formular ciertas inferencias en muestras cuyo tamaño no alcanza el centenar, como es el caso de Aquihuecó. Estos acercamientos se basan en proporciones entre grupos etarios de adultos y subadultos, definidos de manera tal que se eviten sesgos comunes (i.e. menor preservación de infantiles y subestimación de adultos mayores) y permiten evaluar tendencias demográficas (BocquetAppel y Masset 1977).

Los gráficos y análisis se hicieron con el programa $\mathrm{R}$ versión 3.4.1 (R Core Team 2018).

\section{Resultados}

Los análisis permitieron estimar un $\mathrm{NMI}=64$ entre adultos y subadultos. En el caso de los adultos los elementos o zonas diagnósticas más representadas fueron el mastoideo izquierdo $(n=40)$ y la epífisis distal del húmero derecho $(n=40)$. En el caso de los subadultos la porción más representada fue la epífisis proximal del fémur izquierdo $(n=24)$. De este modo, la categoría adulto está representada por el $62,5 \%$, mientras que la categoría subadulto por el $37,5 \%$ (Tabla 1).

De los 64 individuos estimados, $43(67,18 \%)$ fueron hallados in situ, es decir, corresponden a individuos enterrados recuperados en excavaciones sistemáticas. Los restantes se computaron a partir de hallazgos de huesos o fragmentos sueltos que quedaron expuestos y se recolectaron en superficie. En la Tabla 2 se presenta la información bioarqueológica general obtenida hasta el momento de los 43 entierros primarios. Entre los adultos hubo cinco casos que no pudieron ubicarse en una categoría específica debido a la mala preservación de las estructuras diagnósticas, por lo cual fueron considerados como una categoría separada, denominada adulto indeterminado. No se encontraron diferencias en la representación de femeninos y masculinos en las categorías de edad (adultos vs. subadultos) (prueba exacta de Fischer, p=1) (Figura 4). Los indeterminados no fueron incluidos en este análisis. 
Tabla 1. Frecuencia de elementos óseos del sitio Aquihuecó considerados para la estimación del NMI por edad (adultos y subadultos) y lateralidad (derecho e izquierdo). Ref.: px: proximal, ds: distal, md: medial, lat: lateral, Der.: derecho, Izq.: izquierdo; Ax.: axial. En negritas se señalan los elementos más frecuentes.

Frequency of bone elements from the Aquihuecó site used to estimate MNI by age (adults and subadults) and laterality (right and left). Ref.: px: proximal, ds: distal, md: medial, lat: lateral, Der:: right, Izq., left, Ax.: axial. In bold, the most frequent elements.

\begin{tabular}{|c|c|c|c|c|c|c|}
\hline & \multicolumn{3}{|c|}{ Adultos } & \multicolumn{3}{|c|}{ Subadultos } \\
\hline & Der. & Izq. & Ax. & Der. & Izq. & Ax. \\
\hline Mastoideo & 33 & 40 & - & 13 & 10 & - \\
\hline Húmero px & 18 & 21 & - & 7 & 11 & - \\
\hline Húmero ds & 40 & 39 & - & 11 & 16 & - \\
\hline Cúbito px & 35 & 36 & - & 13 & 14 & - \\
\hline Cúbito ds & 19 & 24 & - & 7 & 9 & - \\
\hline Radio px & 32 & 30 & - & 13 & 13 & - \\
\hline Radio ds & 27 & 31 & - & 10 & 9 & - \\
\hline Clavícula md & 21 & 19 & - & 8 & 3 & - \\
\hline Clavícula lat & 28 & 24 & - & 13 & 5 & - \\
\hline Escápula & 26 & 28 & - & 14 & 9 & - \\
\hline Fémur px & 35 & 37 & - & 20 & 24 & - \\
\hline Fémur ds & 34 & 34 & - & 15 & 15 & - \\
\hline Tibia px & 29 & 29 & - & 10 & 16 & - \\
\hline Tibia ds & 26 & 27 & - & 9 & 10 & - \\
\hline Peroné px & 18 & 20 & - & 7 & 6 & - \\
\hline Peroné ds & 25 & 26 & - & 5 & 5 & - \\
\hline Rótula & 18 & 27 & - & 1 & 0 & - \\
\hline Calcáneo & 32 & 19 & - & 4 & 3 & - \\
\hline Astrágalo & 25 & 25 & - & 2 & 2 & - \\
\hline Ilion & 25 & 26 & - & 10 & 15 & - \\
\hline Isquion & 26 & 28 & - & 9 & 9 & - \\
\hline Pubis & 18 & 15 & - & 4 & 6 & - \\
\hline Mandíbula & - & - & 35 & - & - & 12 \\
\hline Atlas & - & - & 14 & - & - & 2 \\
\hline Axis & - & - & 13 & - & - & 3 \\
\hline Cráneo & - & - & 23 & - & - & 15 \\
\hline
\end{tabular}

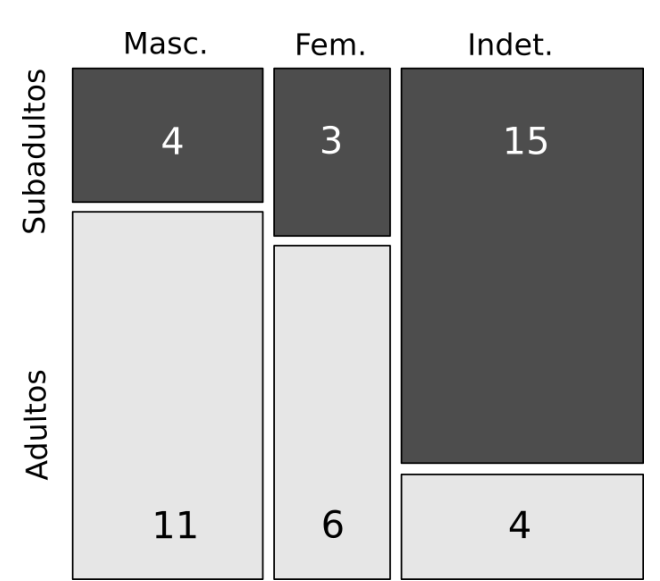

Figura 4. Gráfico de mosaico que muestra la distribución por sexo y categorías de edad (adultos y subadultos) de los individuos hallados in situ ( $\mathrm{n}=43)$.

Mosaic plot showing the distribution by sex and age categories (adult and subadult) of the individuals found in situ ( $n=43$ ). 
Tabla 2. Información bioarqueológica general de los 43 individuos hallados in situ. Ref.: Ent.: entierro, Conj.: conjunto, Ind.: individuo, M: masculino, F: femenino, I: indeterminado (rasgos dimórficos ambiguos), ND: no determinado (por falta de estructuras diagnósticas), C: circular. * Perinato: no-nato a $<1$ mes post-natal (tomado de García Guraieb 2010).

General bioarchaeological information from the 43 individuals found in situ. Ref.: Ent.: Burial; Conj: set; Ind: individual; M: male, F: female, I: indeterminate (ambiguous dimorphic traits), ND: not determined (lack of diagnostic structures), C: circular. *Perinate: unborn up to <1 month post-natal (from García Guraieb 2010).

\begin{tabular}{|c|c|c|c|c|c|c|c|c|}
\hline $\mathrm{N}^{\circ}$ ind. & Denominación & Sexo & Rango de edad (en años) & $\begin{array}{c}\text { Modificación } \\
\text { artificial del cráneo }\end{array}$ & ${ }^{14} \mathrm{C}$ & $\begin{array}{l}\delta^{13} \mathrm{C}_{\mathrm{col}} \\
(\%)\end{array}$ & $\begin{array}{l}\delta^{15} \mathrm{~N} \\
(\%)\end{array}$ & Referencias \\
\hline 1 & Ent. I & $\mathrm{M}$ & $11 \pm 1$ & & & & & \\
\hline 2 & Ent. II & I & $8,5 \pm 2,5$ & & & & & \\
\hline 3 & Ent. III & $\mathrm{M}$ & Adulto & & & & & \\
\hline 4 & Ent. IV.1 & ND & Adulto & & & & & \\
\hline 5 & Ent. IV.2 & ND & $0,25 \pm 0,25$ & & & & & \\
\hline 6 & Ent. V.1 & ND & $\sim 14$ & & & & & \\
\hline 7 & Ent. V.2 & ND & $\sim 8$ & & & & & \\
\hline 8 & Ent. V.3 & ND & $4 \pm 1$ & & & & & \\
\hline 9 & Ent. VI & I & Adulto & & & & & \\
\hline 10 & Ent. VII & M & $42 \pm 7$ & $\mathrm{C}$ & & & & \\
\hline 11 & Ent. 15 & ND & $0,75 \pm 0,25$ & & & & & \\
\hline 12 & Ent. 16.1 & $\mathrm{~F}$ & $42 \pm 7$ & & $3817 \pm 59$ & $-17,6$ & 7,5 & Lema et al 2012; Gordón et al. 2018 \\
\hline 13 & Ent. 16.2 & $\mathrm{ND}$ & Perinato* & & & & & \\
\hline 14 & Ent. 17 & ND & $0,62 \pm 0,1$ & & & & & \\
\hline 15 & Ent. 18 & ND & Perinato* & & & & & \\
\hline 16 & Ent. 19.1 & $\mathrm{~F}$ & $21 \pm 2$ & & & $-18,2$ & 7,5 & Gordón et al. 2018 \\
\hline 17 & Ent. 19.2 & $\mathrm{~F}$ & $39,5 \pm 5,5$ & & & -18 & 8,2 & Gordón et al. 2018 \\
\hline 18 & Ent. 20 & M & $27 \pm 2$ & & & & & \\
\hline 19 & Ent. 21 & $\mathrm{~F}$ & $21 \pm 3$ & & & & & \\
\hline 20 & Ent. 22 & I & $14 \pm 1$ & & $4050 \pm 61$ & & & Lema et al. 2012 \\
\hline 21 & Ent. 23 & $\mathrm{M}$ & $>50$ & $\mathrm{C}$ & $4172 \pm 55$ & $-17,9$ & 10,2 & Lema et al. 2012; Górdon et al. 2018 \\
\hline 22 & Ent. 28 & I & $25,5 \pm 4,5$ & & & & & \\
\hline 23 & Ent. 29 & I & $0,08 \pm 0,08$ & & & & & \\
\hline 24 & Ent. 30 & I & $5 \pm 0,5$ & & & & & \\
\hline 25 & Ind. 24 & $\mathrm{~F}$ & $32 \pm 2$ & & & & & \\
\hline 26 & Ind. 26 & $\mathrm{M}$ & $11 \pm 1$ & & & -18 & 7,4 & Gordón et al. 2018 \\
\hline 27 & Ind. 27 & I & $21 \pm 3$ & $\mathrm{C}$ & $4280 \pm 59$ & -18 & 8,2 & Gordón et al. 2019, 2018 \\
\hline 28 & Ind. 30 & $\mathrm{M}$ & Adulto & $\mathrm{C}$ & & $-17,9$ & 10 & Gordón et al. 2018 \\
\hline 29 & Ind. 31 & $\mathrm{~F}$ & $22 \pm 2$ & & & $-18,1$ & 7,3 & Gordón et al. 2018 \\
\hline 30 & Ind. 32 & $\mathrm{~F}$ & $6 \pm 1$ & & & & & \\
\hline 31 & Ind. 33 & M & $26,5 \pm 1,5$ & & $4045 \pm 66$ & $-18,4$ & 7,1 & Gordón et al. 2019, 2018 \\
\hline 32 & Ind. 34 & M & $32 \pm 2$ & $\mathrm{C}$ & $4304 \pm 59$ & $-18,3$ & 10,4 & Gordón et al. 2019, 2018 \\
\hline 33 & Ind. 35 & M & $5,5 \pm 1,5$ & $\mathrm{C}$ & & & & \\
\hline 34 & Ind. 36 & $\mathrm{~F}$ & $3,5 \pm 0,5$ & & & & & \\
\hline 35 & Ind. 37 & M & $6 \pm 1$ & & & & & \\
\hline 36 & Ind. 38 & M & $37 \pm 2$ & $\mathrm{C}$ & & $-17,9$ & 9,9 & Gordón et al. 2018 \\
\hline 37 & Ind. 39 & I & $\sim 3$ & $\mathrm{C}$ & & & & \\
\hline 38 & Ind. 40 & $\mathrm{M}$ & $28,5 \pm 1,5$ & $\mathrm{C}$ & $4165 \pm 64$ & $-18,3$ & 8,5 & Gordón et al. 2019, 2018 \\
\hline 39 & Conj.2.1 & $\mathrm{F}$ & $11 \pm 1$ & $\mathrm{C}$ & & $-17,8$ & 8 & Gordón et al. 2018 \\
\hline 40 & Conj2.2 & I & $6 \pm 1$ & $\mathrm{C}$ & & & & \\
\hline 41 & Ind. 41 & $\mathrm{M}$ & $26,5 \pm 7,5$ & $\mathrm{C}$ & & & & \\
\hline 42 & Ind. 42 & I & $10 \pm 2$ & & & & & \\
\hline 43 & Ind. 43 & $\mathrm{M}$ & Adulto & $\mathrm{C}$ & & & & \\
\hline
\end{tabular}


En la Tabla 3 se presentan los conteos de individuosenterrados $(n=43$ ) agrupados por categorías de edad y sexo. Sobre esta base, y repartiendo los adultos indeterminados proporcionalmente entre las demás categorías, en la Figura 5 se presenta la curva de mortalidad de Aquihuecó.

Hasta los cinco años se registra el 20,93\% de las muertes, de las cuales el $13,95 \%$ corresponde al primer año de vida. Luego el porcentaje disminuye en las sucesivas categorías de edad y en el rango de 15-19,9 años no se registraron individuos. Entre los adultos el pico se da entre los 25-29,9 años (11,63\%) y luego desciende notablemente en las siguientes categorías.

Respecto a los estimadores paleodemográficos, el índice JAR arrojó un valor de 0,619 , mientras que MCM es de 0,159. En la Tabla 4 se muestran los valores sobre los que se calcularon estos índices.

\section{Discusión}

\section{Paleodemografía, tafonomía y el contexto sedimentológico de Aquihuecó}

Existen diversos factores que median entre una población viva y la muestra osteológica recuperada (Waldron 1994). Estos pueden agruparse en factores intrínsecos (i.e. patrones de mortalidad, fecundidad, morbilidad, condicionantes naturales y sociales) y extrínsecos (i.e. prácticas mortuorias, factores postdepositacionales, diseños de investigación, métodos y técnicas disponibles) (Barrientos 1997). Asimismo, el efecto del tiempo es un factor que incide en la formación de las muestras promediando temporalmente los patrones de mortalidad y fecundidad, que pueden enmascarar procesos de corto plazo (Waldron 1994).

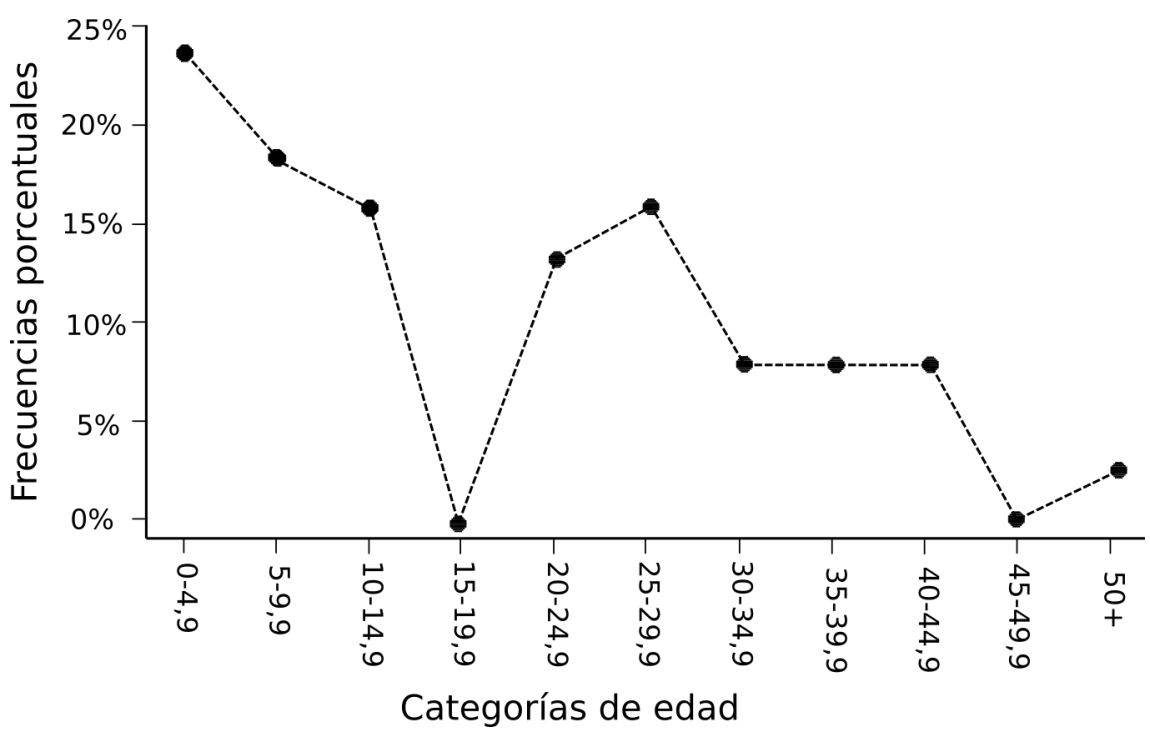

Figura 5. Curva de mortalidad de Aquihuecó ( $\mathrm{n}=43$ ) por rangos de 5 años.

Aquihuecó mortality curve $(n=43)$ in 5-year ranges. 
Tabla 3. Frecuencias absolutas y porcentuales por edad (rangos de 5 años) y sexo. Ref.: M: masculino, F: femenino,

I: indeterminado (rasgos dimórficos ambiguos), ND: no determinado (falta de estructuras diagnósticas), T: total, Ad. I: adulto indeterminado.

Absolute and percentage frequencies by age (5-year ranges) and sex. Ref.: M: male, F: female, I: indeterminate (ambiguous dimorphic traits), ND: not determined (lack of diagnostic structures), T: total, Ad. I: indeterminate adult.

\begin{tabular}{cccccc}
\hline Edad & M & F & I + ND & T & $\%$ \\
\hline $0-4,9$ & 0 & 1 & 8 & 9 & 20,93 \\
$5-9,9$ & 2 & 1 & 4 & 7 & 16,28 \\
$10-14,9$ & 2 & 1 & 3 & 6 & 13,95 \\
$15-19,9$ & 0 & 0 & 0 & 0 & 0,00 \\
$20-24,9$ & 0 & 3 & 1 & 4 & 9,30 \\
$25-29,9$ & 4 & 0 & 1 & 5 & 11,63 \\
$30-34,9$ & 1 & 1 & 0 & 2 & 4,65 \\
$35-39,9$ & 1 & 1 & 0 & 2 & 4,65 \\
$40-44,9$ & 1 & 1 & 0 & 2 & 4,65 \\
$45-49,9$ & 0 & 0 & 0 & 0 & 0,00 \\
$50+$ & 1 & 0 & 0 & 1 & 2,33 \\
Ad. I & 3 & 0 & 2 & 5 & 11,63 \\
Total & 15 & 9 & 19 & 43 & 100,00 \\
\hline
\end{tabular}

Tabla 4. Tabla de vida abreviada para el sitio Aquihuecó. Sobre estos valores se estimaron los parámetros paleodemográficos

JAR y MCM. Ref.: Ad. I: adulto indeterminado.

Abbreviated life table for the Aquihuecó site. These data were used to estimate JAR and MCM values. Ref.: Ad. I: indeterminate adult.

\begin{tabular}{ccccc}
\hline $\begin{array}{c}\text { Rangos } \\
\text { de edad } \\
\text { en años }\end{array}$ & $\begin{array}{c}\mathrm{N}^{\circ} \\
\text { Ind. }\end{array}$ & $\begin{array}{c}\% \\
\text { muertes }\end{array}$ & $\begin{array}{c}\text { Índice de } \\
\text { supervivencia }\end{array}$ & $\begin{array}{c}\text { Probabilidad } \\
\text { de muerte } \mathrm{q}(\mathrm{x})\end{array}$ \\
\hline $0-4,9$ & 9 & 23,68 & 100 & 0,236 \\
$5-9,9$ & 7 & 18,42 & 76,32 & 0,206 \\
$10-14,9$ & 6 & 15,79 & 57,9 & 0,272 \\
$15-19,9$ & 0 & 0,00 & 42,12 & 0 \\
$20-24,9$ & 5 & 13,16 & 42,12 & 0,249 \\
$25-29,9$ & 6 & 15,79 & 31,6 & 0,416 \\
$30-34,9$ & 3 & 7,89 & 18,45 & 0,285 \\
$35-39,9$ & 3 & 7,89 & 13,19 & 0,398 \\
$40-44,9$ & 3 & 7,89 & 7,93 & 0,663 \\
$45-49,9$ & 0 & 0,00 & 2,67 & 0 \\
$50+$ & 1 & 2,63 & 2,67 & 0,985 \\
\hline
\end{tabular}

De este modo, el patrón de mortalidad de una muestra arqueológica probablemente difiera de la estructura de la población.

Pennington (1996, ver discusión en GarcíaGuraieb 2010) ha sugerido que los requerimientos metodológicos indican que ciertos cálculos demográficos pueden realizarse sobre la base de al menos un centenar de individuos. Sin embargo, dado que varios sitios de entierro cuentan con un número menor, las muestras suelen analizarse desde una perspectiva paleodemográfica en términos exploratorios. Aquihuecó, emplazado en un médano de origen eólico, está expuesto a los fuertes vientos imperantes en la región. La estructura que contiene a los entierros es un médano vivo que, si bien se encuentra consolidado, no está litificado, por lo tanto, se trata de una estructura dinámica y activa. La cantidad variable de restos óseos desarticulados y expuestos, los tipos de huesos (i.e. desde pequeños fragmentos hasta huesos completos) y los diversos grados de meteorización, evidencian períodos variables de exposición (sensu Behrensmeyer 1978) (Figura 3).

Esta característica de la dinámica ambiental local impactó sobre el conjunto enterrado. De los 64 individuos estimados, únicamente 43 se recuperaron in situ (22 subadultos y 21 adultos). Esta relación es diferente entre los individuos de superficie (dos subadultos y 19 adultos). De estas observaciones se infiere que la submuestra de superficie estaría sesgada en contra de individuos subadultos. Esto podría vincularse con el transporte y/o deterioro de los restos expuestos. Los restos de subadultos, más livianos, pueden ser transportados por el viento a mayores distancias. Además, tienen menor densidad ósea, lo que los hace más susceptibles al deterioro (Gordon y Buikstra 1981).

Por lo expuesto, se considera la curva de mortalidad y ciertos parámetros en términos descriptivos y exploratorios sobre la estructura sexoetaria de Aquihuecó en base al conjunto de individuos enterrados $(n=43)$. No obstante, ésta muestra presenta características que la convierten en una serie bioarqueológica relevante acotada temporal $(4304 \pm 59$ y $3650 \pm 70$ años AP) y espacialmente ( $18 \mathrm{~m} \mathrm{x} 35$ $\mathrm{m}$, aproximadamente), tanto para las problemáticas demográficas patagónicas en general como para las del sector septentrional de la región en particular.

\section{Características paleodemográficas de Aquihuecó en el contexto regional}

La estimación del NMI incluyó a todos los subadultos en un mismo conjunto; sin embargo, un estudio que contemple en detalle indicadores bioarqueológicos específicos en la muestra de superficie, tales como la longitud de los huesos largos, podría elevar la frecuencia calculada. La curva de Aquihuecó es concordante con el patrón bimodal observado en los perfiles atricionales o acumulativos, en contraposición a aquellos producto de eventos catastróficos, como epidemias o catástrofes naturales (Chamberlain 2006). Este último tipo de perfil refleja 
la estructura de una población viva ya que todos los individuos, independientemente del sexo, edad y estado de salud tienen la misma probabilidad de morir. En un perfil atricional se observa una mortalidad elevada durante el primer año de vida que va disminuyendo hacia los cinco años para llegar al mínimo entre los 10 años y la adultez, momento en el cual comienza a incrementarse (Chamberlain 2006; Gage 2000). La baja frecuencia de individuos $>50$ años $(\mathrm{n}=1)$, ha sido explicada por diversos autores como el producto de sesgos vinculados a la baja sensibilidad de los métodos para estimar la edad en categorías que estén por encima de esta edad biológica (Hoppa y Vaupel 2002).

Un tema central para las reconstrucciones paleodemográficas se refiere a la asignación del sexo en subadultos y, sobre todo, de la edad en adultos (Lewis 2007; Luna 2008; Scheur y Black 2000). De los 22 subadultos únicamente siete pudieron ser asignados a un sexo mientras que los 15 restantes son indeterminados debido a la falta de estructuras diagnósticas y en particular al alto grado de ambigüedad en las técnicas para estimar sexo en subadultos. Entre los adultos, seis individuos se asignaron a la categoría femenino, 11 a masculino y cuatro son indeterminados. En todos los casos el tamaño de las submuestras se reduce y las inferencias estadísticas derivadas plantean incertidumbre.

Los índices JAR y MCM usados en conjunto permiten distinguir situaciones acerca de la dinámica de la población de la cual deriva una muestra. El análisis de ambos estimadores permite hipotetizar la ocurrencia de poblaciones en crecimiento, decrecimiento o estacionarias, o la presencia de sesgos (Bernal et al. 2004; García Guraieb 2010; Jackes 1992). No obstante, en este trabajo se utiliza como un acercamiento a la muestra de Aquihuecó que si bien tiene implicancias importantes para el noroeste patagónico, cuenta con un tamaño absoluto que no admite procedimientos paleodemográficos complejos. Los valores sugeridos por Jackes (2011) por debajo de los cuales se puede evaluar si una población se encuentra en un estado estacionario, creciendo o decreciendo son 0,38 para el índice JAR y 0,135 en el caso de MCM. Valores por encima de estos umbrales indicarían que se está ante muestras sesgadas en alguno de los siguientes aspectos: excavación parcial del sitio, entierros selectivos y/o tamaño muestral. Con respecto al primer aspecto, la evidencia sugiere que el sitio podría no contener más restos humanos. Si bien el lado oeste del médano no fue completamente excavado, los sondeos realizados no arrojaron resultados positivos. Los altos valores de JAR $(0,619)$ sugieren una muestra sesgada con una subrepresentación de adultos lo que podría vincularse con entierros selectivos o tamaño muestral o modificaciones postdepositacionales (Figura 6). El valor de $\operatorname{MCM}(0,159)$, si bien por encima del umbral, se acerca más a este lo cual permite pensar en una población creciente, idea que será retomada considerando evidencias de otras líneas (i.e. molecular, radiocarbónica, isotópica, zooarqueológica).

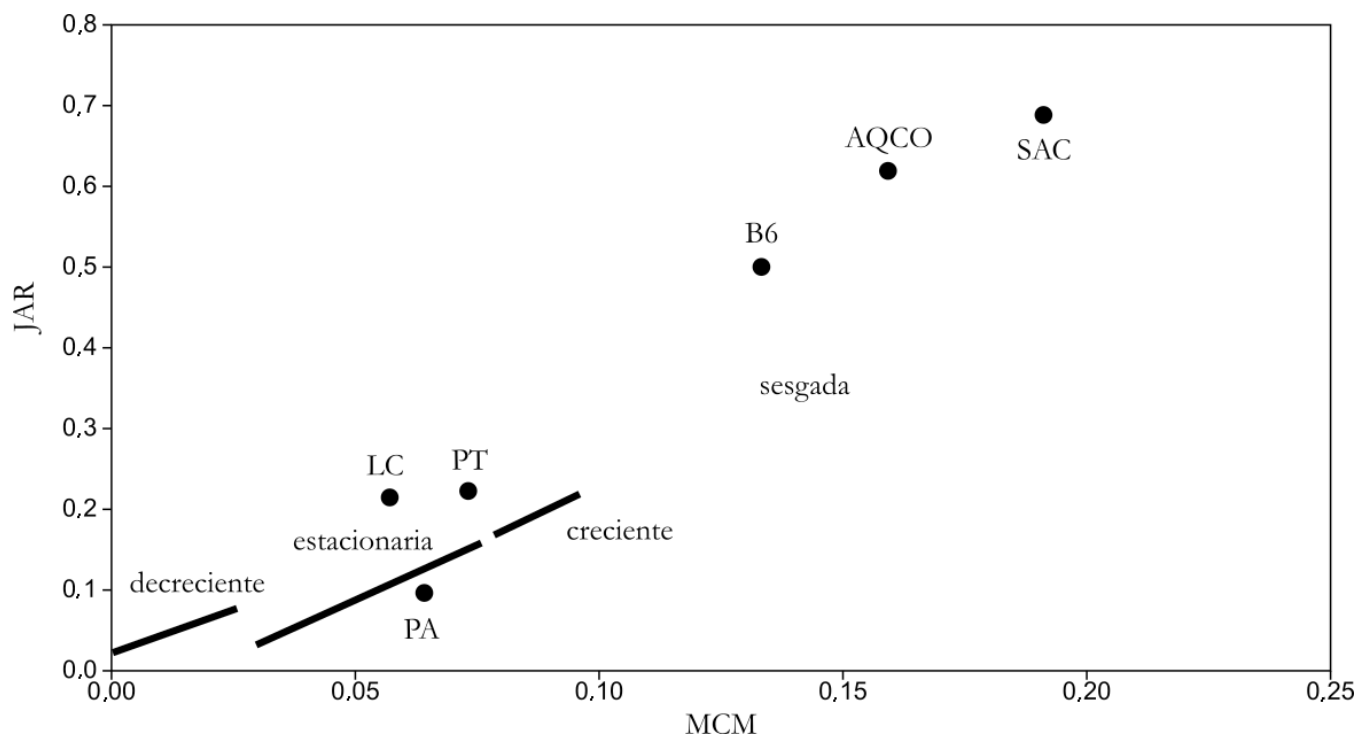

Figura 6. JAR y MCM de Aquihuecó y muestras comparativas. 
En la Tabla 5 se resumen las características generales de las muestras utilizadas para comparar la curva de Aquihuecó. En la Tabla 6 se presentan los datos utilizados para la construcción de las curvas que se muestran en la Figura 7. La curva de Aquihuecó se comporta de manera similar al resto: altos valores de mortalidad en subadultos pequeños, baja en subadultos mayores y elevada desde adultos jóvenes para luego decrecer (curva en forma de U, Jackes 2011). Sin embargo, como se observa en la Tabla 7, los valores del índice de correlación por rangos de Spearman, muestran que Aquihuecó no presenta correlaciones significativas con las demás curvas de mortalidad. Este resultado indica que las frecuencias porcentuales por rangos de edad no se parecen estadísticamente a estos sitios. Asimismo, se compararon los índices JAR y MCM (Tabla 8 y Figura 6). Al igual que SAC y B6, Aquihuecó se encuentra en la zona de muestras sesgadas.

Si bien una serie de factores podrían introducir los sesgos (p.ej., tamaño muestral y preservación diferencial), los resultados obtenidos en Aquihuecó permiten pensar en una población creciente, lo que encuentra sustento en otras líneas de evidencia. Bernal y colaboradores (2018), exploraron los cambios en el tamaño poblacional y la variación en la composición arqueofaunística en el NO de Patagonia, teniendo en cuenta el proceso de extinción de megafauna que ocurrió desde 10.000-8000 años AP. Los autores

Tabla 5. Características generales de los sitios comparativos: ubicación geográfica y cronológica, frecuencia de individuos y subsistencia.

General characteristics of comparative samples. Geographic location, chronology, sample size and subsistence pattern.

\begin{tabular}{|c|c|c|c|c|c|}
\hline Sitio & Ubicación & $\begin{array}{c}\text { Cronología (años } \\
\text { AP) }\end{array}$ & $\begin{array}{l}\mathrm{N}^{\circ} \\
\text { Ind. }\end{array}$ & Subsistencia & Referencias \\
\hline Punta Teatinos & $\begin{array}{l}\text { Bahía de Coquimbo, norte } \\
\text { semi-árido de Chile }\end{array}$ & $4905-1920$ & 202 & Pescadores & Quevedo et al. 2000 \\
\hline B6 & $\begin{array}{c}\text { Margen Río Mendoza. Dto. } \\
\text { Maipú. }\end{array}$ & $2180-2340$ & 34 & $\begin{array}{l}\text { Cazadores } \\
\text { recolectores }\end{array}$ & Novellino et al. 2013 \\
\hline $\begin{array}{l}\text { Lihué Calel - } \\
\text { El Chenque I }\end{array}$ & $\begin{array}{c}\text { Parque Nacional Lihué Calel, } \\
\text { Pampa Seca }\end{array}$ & $1030-370$ & 216 & $\begin{array}{l}\text { Cazadores } \\
\text { recolectores }\end{array}$ & Luna 2008, 2012 \\
\hline Paso Alsina I & $\begin{array}{l}\text { Curso inferior del río Colorado, } \\
\text { transición Pampa-Patagonia }\end{array}$ & $500-450$ & 77 & $\begin{array}{l}\text { Cazadores } \\
\text { recolectores }\end{array}$ & Flensborg 2012 \\
\hline $\begin{array}{c}\text { Sierra } \\
\text { Colorada }\end{array}$ & Nor-oeste de Santa Cruz & $800-350$ & 71 & $\begin{array}{l}\text { Cazadores } \\
\text { recolectores }\end{array}$ & García Guraieb et al. 2015 \\
\hline
\end{tabular}

Tabla 6. Frecuencias absolutas y porcentuales de individuos de Aquihuecó y sitios comparativos por categorías de edad (rangos de 5 años en subadultos y de 15 en adultos). Ref.: Aqco: Aquihuecó, SAC: Sierra Colorada, PT: Punta Teatinos, PA: Paso Alsina I, LC: Lihué Calel, sitio El Chenque I.

Absolute and percentage frequencies of individuals by age ranges (5-year ranges for subadults and 15-year ranges for adults). Aquihuecó and comparative sites. Ref:: Aqco: Aquihuecó, SAC: Sierra Colorada, PT: Punta Teatinos, PA: Paso Alsina I, LC: Lihué Calel, El Chenque I.

\begin{tabular}{ccccccccccccc}
\hline & Aqco & & SAC & \multicolumn{3}{c}{ B6 } & & PT & & PA & \multicolumn{3}{c}{ LC } \\
\hline & $\mathrm{N}$ & $\%$ & $\mathrm{~N}$ & $\%$ & $\mathrm{~N}$ & $\%$ & $\mathrm{~N}$ & $\%$ & $\mathrm{~N}$ & $\%$ & $\mathrm{~N}$ & $\%$ \\
\hline $5-4,9$ & 9 & 20,93 & 16 & 22,54 & 6 & 17,65 & 37 & 18,32 & 11 & 14,29 & 55 & 25,46 \\
$5-9,9$ & 7 & 16,28 & 13 & 18,31 & 3 & 8,82 & 22 & 10,89 & 2 & 2,60 & 18 & 8,33 \\
$10-14,9$ & 6 & 13,95 & 7 & 9,86 & 6 & 17,65 & 7 & 3,47 & 3 & 3,90 & 7 & 3,24 \\
$15-19,9$ & 0 & 0,00 & 6 & 8,45 & 1 & 2,94 & 5 & 2,48 & 7 & 9,09 & 20 & 9,26 \\
$20-34,9$ & 15 & 34,88 & 10 & 14,08 & 3 & 8,82 & 75 & 37,13 & 37 & 48,05 & 65 & 30,09 \\
$35-49,9$ & 5 & 11,63 & 15 & 21,13 & 13 & 38,24 & 56 & 27,72 & 16 & 20,78 & 41 & 18,98 \\
$50+$ & 1 & 2,33 & 4 & 5,63 & 2 & 5,88 & 0 & 0 & 1 & 1,30 & 10 & 4,63 \\
Total & 43 & 100 & 71 & 100 & 34 & 100 & 202 & 100 & 77 & 100 & 216 & 100 \\
\hline
\end{tabular}




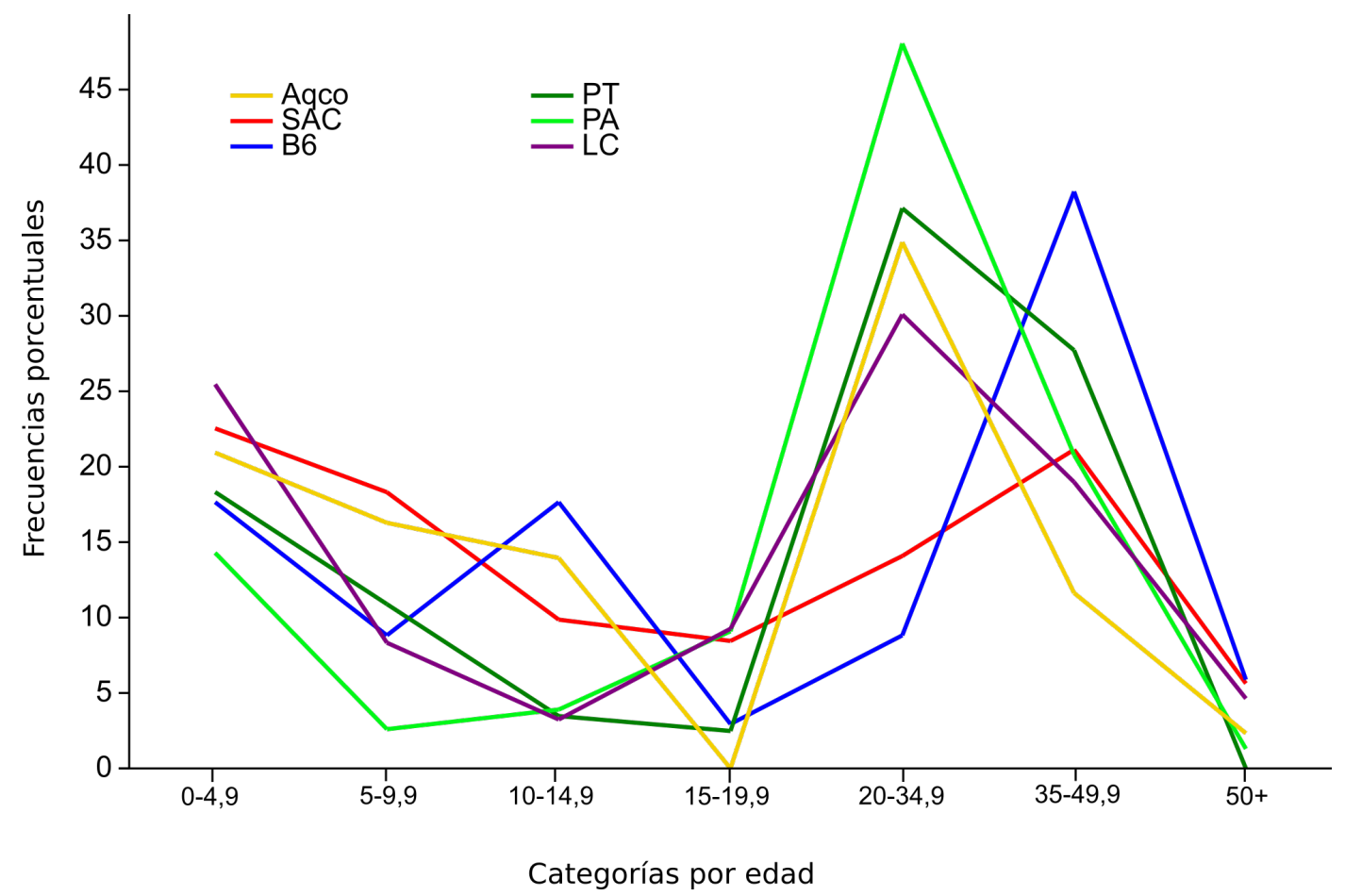

Figura 7. Curvas de mortalidad: Aquihuecó y muestras comparativas.

Mortality curves: Aquihuecó and comparative samples.

Tabla 7 Análisis de correlación de la distribución de edades entre sitios. En la parte inferior izquierda se observan valores de los índices de correlación por rangos de Spearman (rs.). En la parte superior derecha se indican las probabilidades (p) de las pruebas de significación de las correlaciones (nivel de significación <0,05). Ref.: Aqco: Aquihuecó, SAC: Sierra Colorada, PT: Punta Teatinos, PA: Paso Alsina I, LC: Lihué Calel, El Chenque I.

Correlation analysis of the distribution of ages between sites. In the lower left part, the values of correlation indices by Spearman ranges ( $r s$.) are observed. The probabilities ( $p$ ) of the significance tests of the correlations are indicated in the upper right part (significance level <0.05). Ref.: Aqco: Aquihuecó, SAC: Sierra Colorada, PT: Punta Teatinos, PA:

Paso Alsina I, LC: Lihué Calel, El Chenque I.

\begin{tabular}{lrrrrrr}
\hline & Aqco & SAC & B6 & PT & PA & LC \\
\hline Aqco & & 0,139 & 0,375 & 0,066 & 0,302 & 0,236 \\
SAC & 0,607 & & 0,062 & 0,066 & 0,236 & 0,167 \\
B6 & 0,400 & 0,746 & & 0,165 & 0,354 & 0,730 \\
PT & 0,750 & 0,750 & 0,600 & & 0,012 & 0,048 \\
PA & 0,464 & 0,536 & 0,418 & 0,857 & & 0,012 \\
LC & 0,536 & 0,571 & 0,164 & 0,786 & 0,857 & \\
\hline
\end{tabular}

Tabla 8. Índices JAR y MCM por sitios. Ref.: Aqco: Aquihuecó, SAC: Sierra Colorada, PT: Punta Teatinos, PA: Paso Alsina I, LC: Lihué Calel, El Chenque I.

JAR and MCM indices by sites. Ref.: Aqco: Aquihuecó, SAC: Sierra Colorada, PT: Punta Teatinos, PA: Paso Alsina I, LC: Lihué Calel, El Chenque I.

\begin{tabular}{lcc}
\hline & JAR & MCM \\
\hline Aqco & 0,619 & 0,159 \\
SAC & 0,689 & 0,191 \\
B6 & 0,500 & 0,133 \\
PT & 0,221 & 0,073 \\
PA & 0,094 & 0,064 \\
LC & 0,215 & 0,057 \\
\hline
\end{tabular}

analizaron datos moleculares (ADNmt) modernos y antiguos y datos zooarqueológicos desde el Pleistoceno final hasta el Holoceno tardío observando que el número de especies disponibles decrece a lo largo del tiempo y la biomasa consumida se incrementa. Tal relación inversa sugiere que los grupos consumieron 
un número cada vez menor de especies y que esas especies fueron cada vez más explotadas. A partir de datos moleculares estimaron una tendencia positiva al incremento sostenido del tamaño de las poblaciones humanas. En el NO de Patagonia la tasa de crecimiento poblacional se habría acelerado después de 7000-6000 años AP. Asimismo, el análisis estadístico robusto de la frecuencia y distribución de fechados radiocarbónicos condujo a estimaciones demográficas similares. Las curvas muestran para toda la región patagónica que, si bien las poblaciones se habrían incrementado durante el Holoceno, este aumento se habría dado de manera acelerada luego del rango 7000-5000 años AP para alcanzar su tamaño máximo alrededor del 1000 AP. Para el Holoceno tardío estimaron una densidad entre cinco y 20 individuos $/ 100 \mathrm{Km}^{2}$ (Perez et al. 2016).

Los cambios demográficos del Holoceno Tardío han sido vinculados, principalmente, a cambios tecnológicos como el uso de piedras de moler registradas hacia fines del Holoceno Medio (5000-4000 años AP) (Della Negra et al. 2014; Lema et al. 2012; Perez et al. 2009) y cerámica alrededor de 1900 años AP (Della Negra 2008), antes de que las poblaciones alcanzaran su tamaño máximo. Este tipo de innovaciones culturales asociadas al procesamiento e intensificación en el uso de determinados recursos puede haber contribuido al aumento del tamaño poblacional mediante una mayor cantidad de nutrientes potencialmente utilizables (Carmody et al. 2011).

Sobre la base de evidencia paleontológica, arqueofaunística y molecular, Pires et al. (ms) compararon curvas demográficas de poblaciones de Lama guanicoe (guanaco) y humanos en Patagonia. Los autores observan tendencias similares en la trayectoria de ambas curvas: luego de la extinción de la megafauna las poblaciones de guanaco habrían aumentado rápidamente hacia 8000 años $\mathrm{AP}$ alcanzando su pico hace aproximadamente 2500 años. Las poblaciones de cazadores-recolectores de Patagonia habrían aumentado su tamaño luego del 7500 AP estimándose un tamaño efectivo cuatro veces mayor hacia el 5000 AP en comparación con el estimado para la transición Pleistoceno-Holoceno (Pires et al. ms).

Los análisis isotópicos sugieren que la ampliación del nicho de los grupos del NO de Patagonia pudo haberse dado incorporando otros recursos además de los faunísticos. Los valores en colágeno de $\delta^{13} \mathrm{Cy} \delta^{15} \mathrm{~N}$ sugieren el consumo de vegetales desde 7000-6000 años AP (Fernández y Panarello 2001; Gordón et al. 2018), en consonancia con las fechas propuestas en los trabajos previamente mencionados. Sin embargo, los resultados isotópicos muestran cierta heterogeneidad en este patrón, específicamente en la muestra del norte neuquino, cuyo incremento en la densidad poblacional parece no haber estado asociada a un uso intensivo de plantas. Esto podría ser explicado por las condiciones ambientales locales, particularmente por una elevada capacidad de carga del área para las principales presas explotadas por los humanos (Rhea y Lama) (Barberena 2013; Gordón et al. 2018). Asimismo, los movimientos estacionales de los grupos humanos en el norte neuquino siguiendo a las presas habrían contribuido a maximizar la capacidad de sustento del ambiente (Hajduk y Lezcano 2007).

En este contexto, se propone que el sitio Aquihuecó constituye parte de la evidencia empírica del crecimiento poblacional sugerido luego de 70005000 años AP por las diversas líneas de evidencia. Cabe mencionar la presencia del sitio Hermanos Lazcano, ubicado a $21 \mathrm{Km}$ de Aquihuecó. Se trata de un sitio exclusivo de entierro que incluye inhumaciones múltiples con alta concentración de individuos $(\mathrm{n}=12)$ en un área de $9 \mathrm{~m}^{2}$. Hermanos Lazcano comparte una serie de características con Aquihuecó: ambos se emplazan en un sector elevado de la topografía, presentan una cronología similar (Della Negra et al. 2014; Gordón et al. 2019) y características culturales (i.e. deformación artificial del cráneo, bases de molinos, cuentas y colgantes de valva, esferas líticas y litos de fractura trapezoidal). En un trabajo previo, se señaló que el conjunto de estas características compartidas permite sugerir cambios organizacionales que se habrían extendido entre los grupos del norte neuquino durante la transición Holoceno Medio-Tardío (Della Negra et al. 2014). Las características del registro bioarqueológico del NO de Patagonia señalan que el surgimiento de áreas de entierro con alta concentración de individuos se habría producido en momentos más tempranos que en otras regiones patagónicas, donde los registros más antiguos no exceden los 3000 años AP (Bernal et al. 2008; Prates y Di Prado 2013).

\section{Consideraciones Finales}

Este trabajo constituye el primer análisis que integra la totalidad de la muestra de individuos recuperados en el sitio Aquihuecó desde una perspectiva paleodemográfica. Si bien diversos factores pueden haber influido en el perfil paleodemográfico estimado, Aquihuecó es un sitio clave para comprender la dinámica poblacional del noroeste de Patagonia durante la transición Holoceno Medio-Tardío. Los resultados obtenidos son concordantes tanto con los escenarios paleodemográficos inferidos en áreas vecinas, como con los resultados alcanzados en el área por otras líneas de evidencia (p.ej., zooarqueología, ADNmt, isótopos estables, fechados radiocarbónicos, cultura material).

Se espera que el aporte de algunas líneas que restan ser indagadas en profundidad, como el estudio paleopatológico detallado y el ajuste del perfil sexo-etario de la muestra, junto con el incremento 
de datos que están siendo generados (i.e. fechados radiocarbónicos, isótopos estables, ecología isotópica, tafonomía, entre otros) aporten herramientas en el corto plazo para avanzar en la discusión de las variaciones en los patrones paleodemográficos en el norte neuquino durante el Holoceno Medio-Tardío desde una perspectiva ecológico-evolutiva.
Agradecimientos: A los editores, evaluadores anónimos y a los Dres. V. Bernal, S. García-Guraieb y D. Rindel por sus comentarios y sugerencias, que de diversas maneras mejoraron el manuscrito. Este trabajo se desarrolló en el marco de los proyectos PIP CONICET- 0428-2011-2013, PICT-Agencia 20142134 y PIP 729- 2015-2017.

\section{Referencias Citadas}

Acsádi, G. y J. Nemeskéri 1970. History of Human Life Span and Mortality. Akadémiai Kiadó, Budapest.

Anderson, D., G. Thompson y F. Popovich 1976. Age of attainment of mineralization stages of the permanent dentition. Journal of Forensic Sciences 21:191-200.

Barberena, R. 2013. Biogeografía, competencia y demarcación simbólica del espacio: modelo arqueológico para el norte de Neuquén. Intersecciones en Antropología 14:367-381.

Barrientos, G. 1997. Nutrición y Dieta de las Poblaciones Aborígenes Prehispánicas del Sudeste de la Región Pampeana. Tesis de Doctorado Inédita, Facultad de Ciencias Naturales, Universidad Nacional de La Plata, La Plata.

Béguelin, M. 2017. Morfología postcraneana de las poblaciones humanas neuquinas del Holoceno tardío. En $\mathrm{El}$ Poblamiento Humano del Norte de Neuquén. Estado Actual del Conocimiento y Perspectivas, editado por F. Gordón, R. Barberena y V. Bernal, pp.167-190. Aspha ediciones, Buenos Aires.

Béguelin, M. y P. González 2008. Estimación del sexo en poblaciones sudamericanas mediante funciones discriminantes para el fémur. Revista Argentina de Antropología Biológica 10 (2):55-70.

Béguelin, M., F. Lotto y P. González 2011. Estimación del sexo en cazadores-recolectores de Sudamérica a partir de variables métricas del húmero. Intersecciones en Antropología 12:61-68.

Behrensmeyer, A. 1978. Taphonomic and ecologic information from bone weathering. Paleobiology 4 (2):150-162.

Bernal, V., S. García Guraieb y P. González 2004. Perfiles de mortalidad de las muestras de restos óseos humanos procedentes del área del lago Salitroso (provincia de santa Cruz). En Contra Viento y Marea. Arqueología de la Patagonia, editado por T. Civalero, P. Fernández y G. Guraieb, pp. 361-373. INAPL-SAA, Buenos Aires.

Bernal, V., P. González, S.I. Perez y H. Pucciarelli 2008. Entierros humanos del noreste de Patagonia: nuevos fechados radiocarbónicos. Magallania 36 (2):125-134.

Bernal, V., S.I. Perez, M.B. Postillone y D. Rindel 2018. Hunter-gatherer persistence and demography in Patagonia (southern South America): the impact of ecological changes during the Pleistocene and Holocene. En Hunter-Gatherer Resilience in Bioarchaeological Perspective. Cambridge. En Prensa.

Bocquet-Appel, J. y C. Masset 1977. Estimateurs en paléodémographie. L'Homme XVIII:65-90.

Bocquet-Appel, J. y C. Masset 1982. Farewell to paleodemography. Journal of Human Evolution 11:321-333.
Brooks, S. y M. Suchey 1990. Skeletal age determination based on the os pubis: a comparison of the Acsàdi y Nemeskéri and Suchey-Brooks Methods. Human Evolution 5:227-238.

Buikstra, J. y D. Ubelaker 1994. Standards for Data Collection from Human Skeletal Remains. Arkansas Archaeological Survey Research. Series $N^{\circ} 44$, Arkansas, U.S.A.

Carmody, R., G. Weintraub y R. Wrangham 2011. Energetic consequences of thermal and nonthermal food processing. Proceedings of the National Academy of Sciences USA 108:19199-19203.

Chamberlain, A.T. 2006. Demography in Archaeology. Cambridge University Press, Cambridge, U.K.

Cobos, V. y V. Bernal 2017. La evolución de la morfología facial de las poblaciones humanas de Neuquén durante el Holoceno medio-tardío. En El Poblamiento Humano del Norte de Neuquén. Estado Actual del Conocimiento y Perspectivas, editado por F. Gordón, R. Barberena y V. Bernal, pp.191-206. Aspha ediciones, Buenos Aires.

Della Negra, C. 2008. Gubevi I: un sitio con restos óseos humanos asociados a cerámica en el departamento de Minas, zona norte de la provincia del Neuquén. Terceras Jornadas de Historia de la Patagonia, San Carlos de Bariloche.

Della Negra, C. y V. Ibáñez Saint-Paul 2012. Adornos personales durante el Holoceno en el Neuquén, su relevancia simbólica. Comechingonia Virtual VI:39-58.

Della Negra, C. y P. Novellino 2005. Aquihuecó: un cementerio arqueológico en el norte de la Patagonia, valle del Curi Leuvú, Neuquén, Argentina. Magallania 33:165-172.

Della Negra, C., P. Novellino, S.I. Perez, A. Hajduk y V. Bernal 2009. Investigaciones arqueológicas y bioarqueológicas en el sitio Aquihuecó (Norpatagonia): nuevos resultados. En Arqueología de Patagonia: una Mirada desde el Último Confín, editado por M. Salemme, F. Santiago, M. Alvarez, E. Piana, M. Vázquez y E. Mansur, tomo 2, pp. 669-676. Editorial Utopías, Ushuaia.

Della Negra, C., P. Novellino, M. Béguelin, F. Gordón, P. Gonzalez, R. Vázquez y V. Bernal 2014. Áreas de entierro en cazadores-recolectores del Noroeste de Patagonia: sitio Hermanos Lazcano (Chos Malal, Neuquén). Revista Runa 35:5-19.

Demirjian, A., H. Goldstein y J. Tanner 1973. A new system of dental age assessment. Human Biology 45 (2):211-227.

Fernández, J. y H. Panarello 2001. Cazadores recolectores del Holoceno medio y superior de la cueva Haichol, región cordillerana central del Neuquén, República Argentina. Relaciones de la Sociedad Argentina de Antropología 26:9-30. 
Flensborg, G. 2012. Análisis paleopatológico en el curso inferior del río Colorado (Pcia. de Buenos Aires). Exploración y evaluación del estado de salud de sociedades cazadoras recolectoras en el Holoceno Tardio. Tesis de Doctorado inédita. Facultad de Ciencias Sociales, Universidad Nacional del Centro de la Provincia de Buenos Aires, Olavarría.

Flensborg, G., G. Martínez y P. Bayala 2015. Mortality profiles of hunter-gatherer societies: a case study from the Eastern Pampa-Patagonia transition (Argentina) during the Final Late Holocene. International Journal of Osteoarchaeology 25:816826.

Gage, T.B. 2000. Demography. En Human Biology: An Evolutionary and Biocultural Perspective., editado por S. Stinson, B. Bogin, R. Huss-Ashmore y D. O'Rourke, pp. 507-551. WileyLiss, New York.

García Guraieb, S. 2010. Bioarqueología de Cazadores-Recolectores del Holoceno Tardío de la Cuenca del Lago Salitroso (Santa Cruz): Aspectos Paleodemográficos y Paleopatológicos. Tesis de Doctorado inédita, Facultad de Filosofía y Letras, Universidad de Buenos Aires, Buenos Aires.

García Guraieb, S., P. González y V. Bernal 2007. Estructura de sexo y edad de la muestra de restos humanos del Holoceno tardío del lago Salitroso (Santa Cruz, Argentina). En Arqueología de Fuego-Patagonia. Levantando Piedras, Desenterrando Huesos y Develando Arcanos, editado por F. Morello, M. Martinic, A. Prieto y G. Bahamonde, pp. 367-374. Ediciones CEQUA, Punta Arenas.

García Guraieb, S., R. Goñi y A. Tessone 2015. Paleodemography of Late Holocene hunter-gatherers from Patagonia (Santa Cruz, Argentina): an approach using multiple archaeological and bioarchaeological indicators. Quaternary International 356:147-158.

Gordon, C. y J. Buikstra 1981. Soil pH, bone preservation, and sampling bias at mortuary sites. American Antiquity 46:566571.

Gordón, F., M. Béguelin, D. Rindel, C. Della Negra, A. Hajduk, R. Vázquez, V. Cobos, S.I. Perez y V. Bernal 2019. Estructura espacial y dinámica temporal de la ocupación humana de Neuquén (Patagonia argentina) durante el Pleistoceno final - Holoceno. Manuscrito en posesión de los autores.

Gordón, F., V. Bernal, M. Béguelin, C. Della Negra y P. Novellino 2013. Bioarqueología del norte de Neuquén. Primera aproximación desde la Teoría de Construcción de Nicho. XVIII Congreso Nacional de Arqueología Argentina, La Rioja.

Gordón, F. y P. Novellino 2017. Patrones paleodietarios en el noroeste de la Patagonia argentina durante el Holoceno tardío: bioindicadores de salud bucal e isótopos estables en restos óseos humanos. En El Poblamiento Humano del Norte del Neuquén: Estado Actual del Conocimiento, editado por F. Gordón, F., R. Barberena y V. Bernal, pp. 145-166. Aspha ediciones, Buenos Aires.

Gordón, F., S.I. Perez, A. Hajduk, M. Lezcano y V. Bernal 2018. Dietary patterns in human populations from Northwest Patagonia during Holocene: an approach using Binford's frames of reference and Bayesian isotope mixing models. Archaeological and Anthropological Sciences 10:1347-1358.

Hajduk, A., P. Novellino, E. Cúneo, A. Albornoz, C. Della Negra y M. Lezcano 2007. Estado de avance de las investigaciones arqueológicas en el noroeste de la provincia del Neuquén (departamentos Chos Malal y Minas, República Argentina) y su proyección futura. En Arqueología de Fuego
Patagonia. Levantando Piedras, Desenterrando huesos y Develando Arcanos, editado por F. Morello, M. Martinic, A. Prieto y G. Bahamonde, pp. 467-478. Editorial CEQUA, Punta Arenas.

Hajduk, A. y M. Lezcano 2007. Entre invernadas y veranadas: prospecciones arqueológicas en la cuenca superior del río CuriLeuvú (norte Neuquino). XVI Congreso Nacional de Arqueología Argentina, San Salvador de Jujuy.

Hajduk, A. y E Vargas 2017. Allende y Aquende. Evidencias arqueológicas compartidas en ambas márgenes andinas Norpatagónicas: arte rupestre, expresiones alfareras, moluscos marinos y piezas metálicas. I Taller de Discusión de Arqueología y Bioantropología del Neuquén, Aluminé.

Hoppa, R. D. 2001. The once and future paleodemography. En Out of the Past. The History of Human Osteology at the University of Toronto, editado por L. Sawchuk y S. Pfeiffer, CITD press, University of Toronto. Scarborough. http://citdpress.utsc.utoronto. ca/osteology/pfeiffer.html

Hoppa, R.D. y J.W. Vaupel 2002. Paleodemography. Age Distributions from Skeletal Samples. Cambridge University Press, Cambridge.

Jackes, M. 1986. The mortality of Ontario archaeological populations. Canadian Journal of Anthropology 5:33-48.

Jackes, M. 1992. Paleodemography: problems and techniques. En Skeletal Biology of Past Peoples: Research Methods, editado por S. Saunders y A. Katzenberg, pp. 189-224. Wiley-Lyss, New York.

Jackes, M. 2011. Representativeness and bias in archaeological skeletal samples. En Social Bioarchaeology, editado por S. Agarwal y B. Glencross, pp. 107-146. Wiley-Blackwell.

Kahana, T., W. Birkby, L. Goldin y J. Hiss 2003. Estimation of age in adolescents- the basilar synchondrosis. Journal of Forensic Science 48 (3):1-5.

Lema. V., C. Della Negra y V. Bernal 2012. Explotación de recursos vegetales silvestres y domesticados en Neuquén: implicancias del hallazgo de restos de maíz y algarrobo en artefactos de molienda del Holoceno tardío. Magallania 40 (1):229-247.

Lewis, M.E. 2007. The Bioarchaeology of Children. Perspectives from Biological and Forensic Anthropology. Cambridge University Press, New York.

Livi-Bacci, M. 1993. Introducción a la Demografía. Ariel, Barcelona, España.

Loth, S. y M. Henneberg 2001. Sexually dimorphic mandibular morphology in the first few years of live. American Journal of Physical Anthropology 115:179-186.

Lovejoy, C., R. Meindl, T. Pryzbeck y R. Mensforth 1985. Chronological methamorphosis of the auricular surface of the ilium: a new method for the determination of adult skeletal age at death. American Journal of Physical Anthropology 68:15-28.

Luna, L. 2008. Estructura Demográfica y Estilo de Vida de Cazadores-Recolectores en un ambiente de desierto. Sitio Chenque I (Parque Nacional Lihué Calel, Provincia de La Pampa). Tesis de Doctorado inédita. Facultad de Filosofía y Letras, Universidad de Buenos Aires, Buenos Aires.

Luna, L. 2012. Validación de métodos para la generación de perfiles de mortalidad a través de la dentición. Su importancia para la caracterización paleodemográfica. Revista Argentina de Antropología Biológica 14:33-51. 
McKern, T, y T. Stewart 1957. Skeletal age changes in young American males, analyzed from the standpoint of identification. Technical Report EP-45. Headquarters, Quartermaster Research and Development Command. Natick, Massachusetts.

Martínez, G. 2017. Conclusiones. En Arqueología de Cazadores Recolectores del Curso Inferior del Río Colorado (provincia de Buenos Aires, Argentina). Aportes al Conocimiento de las Ocupaciones Humanas Pampeano-Patagónicas, editado por G. Martínez, pp. 298-311. INCUAPA CONICET UNICEN, Olavarría.

Martínez, G. y G. Flensborg 2018. Nuevos contextos funerarios en la transición pampeano-patagónica oriental (Pcia. de Buenos Aires, Argentina): aportes al Holoceno Medio y Tardío inicial. Chungara Revista de Antropología Chilena 50 (3):441-458.

Meindl, R.S. y C.O. Lovejoy 1985. Ectocranial suture closure: a revised method for the determination of skeletal age at death based on the lateral-anterior sutures. American Journal of Physical Anthropology 68 (1):57-66.

Meindl, R.S. y C.O. Lovejoy 1989. Age changes in the pelvis: implications for paleodemography. En Age Markers in the Human Skeleton, editado por M.Y. Iscan, pp. 137-168. Springfield IL Charles C Thomas.

Mengoni-Goñalons, G. 1999. Cazadores de Guanacos de la Estepa Patagónica. Sociedad Argentina de Antropología, Buenos Aires.

Milner, R.G., J.W. Wood y J.L. Boldsen 2000. Paleodemography. En Biological Anthropology of the Human Skeleton, editado por M.A. Katzenberg y S.R. Saunders, pp. 467-498. WilleyLiss Inc., New York.

Molleson, T., K. Cruse y S. Mays 1998. Some sexually dimorphic features of the human juvenile skull and their value in sex determination in inmature skeletal remains. Journal of Archaeological Science 25 (8):719-728.

Novellino, P., N. Brachetta-Aporta, L. D'Addona, D. Estrella, V. Bernal, S. Devincenzi, V. Cortegoso y V. Durán 2013. Sitios de entierro de la localidad arqueológica Barrancas, Maipú (Mendoza, Argentina). Intersecciones en Antropología 14:271-277.

Owings Webb, P. y J. Suchey 1985. Ephiphyseal union of the anterior iliac crest and medial clavicle in a modern multiracial sample of American males and females. American Journal of Physical Anthropology 68:457-466.

Pennington, R. 1996. Causes of early human population growth. American Journal of Physical Anthropology 99:259274.

Perez, S.I., C. Della Negra, P. Novellino, P. González, V. Bernal, E. Cúneo y A. Hajduk 2009. Deformaciones artificiales del cráneo en cazadores-recolectores del Holoceno mediotardío del noroeste de Patagonia. Magallania 37 (2):77-90.

Perez, S.I., M.B. Postillone, D. Rindel, D. Gobbo, P. González y V. Bernal 2016. Peopling time, spatial occupation and demography of Late Pleistocene-Holocene human population from Patagonia. Quaternary International 425:214-223.
Phenice, T.W. 1969. A newly development visual method of sexing the os pubis. American Journal of Physical Anthropology 30:297-301.

Pires, M.M., D. Rindel, P. Guimaraes Jr., S.F. dos Reis, S.I. Perez 2016. Human impact on Patagonian megafauna and the consequences of megafaunal extinction for surviving species, ms.

Prates, L. y V. Di Prado 2013. Sitios con entierros humanos y ocupaciones residenciales en la cuenca del río Negro (Norpatagonia, Argentina). Diacronía y multicausalidad. Latin American Antiquity 24 (4):451-466.

Quevedo, S., J. Cocilovo, M. Acosta, H. Varela y S. Valdano 2000. Perfil paleodemográfico de Punta Teatinos, una población de pescadores arcaicos del norte semiárido de Chile. Boletín del Museo Nacional de Historia Natural 49:237-256.

R Core Team 2018. R: A language and environment for statistical computing. R Foundation for Statistical Computing, Vienna, Austria. URL https://www.R-project.org/.

Rindel, D. 2017. Explorando la variabilidad en el registro zooarqueológico de la provincia del Neuquén: tendencias cronológicas y patrones de uso antrópico En El Poblamiento Humano del Norte del Neuquén: Estado Actual del Conocimiento, editado por F. Gordón, R. Barberena y V. Bernal, pp. 101-122. Aspha ediciones, Buenos Aires.

Sattenspiel, L. y H. Harpending 1983. Stable populations and skeletal age. American Antiquity 48:489-497.

Scheuer, L. y S. Black 2000. Developmental Juvenile Osteology, Academic Press, Londres.

Scheuer, L. y S. Black 2004. The Juvenile Skeleton. Elsevier Academic Press, London.

Schulze, D., U. Rother, A. Fuhrmann, S. Richel, G. Faulmann y M. Heiland 2006. Correlation of age and ossification of the medial clavicular epiphysis using computed tomography. Forensic Science International 158:184-189.

Schutkowski, H. 1993. Sex determination of infant and juvenile skeletons: I. Morphognostic features. American Journal of Physical Anthropology 90:199- 205.

Ubelaker, D. 1974. Reconstruction of demographic profiles from ossuary skeletal samples. A case study from the Tidewater Potomac. Smithsonian Contributions to Anthropology 18. Smithsonian Institution Press, Washington DC.

Ubelaker, D. 1982. Human Skeletal Remains. Excavation, Analysis, Interpretation. Taraxacum, Washington DC.

Ubelaker, D. 1989. Human Skeletal Remains. Excavation, Analysis, Interpretation. $2^{\circ}$ Ed. Taraxacum, Washington DC.

Waldron, T. 1994. Counting the Dead: The Epidemiology of Skeletal Populations. John Willey \& Sons, Chichester.

Weaver, D. 1980. Sex differences in the ilia of a known sex and age sample of fetal and infant skeletons. American Journal of Physical Anthropology 52:191-195. 
